\title{
Urticaria, angioedema, and type I hypersensitivity reactions associated with fibrinolytic agents
}

\author{
Kawin Duangmee, ${ }^{1}$ Pornwalai Boonmuang, ${ }^{2}$ Wichai Santimaleeworagun, ${ }^{2}$ Hutsaya Prasitdumrong ${ }^{3}$
}

\begin{abstract}
Background: Several clinical trials of fibrinolytic agents have reported the occurrence of allergic reactions, in addition to hemorrhage. These reactions might worsen patient outcomes, especially by causing life-threatening type I hypersensitivity reactions, including anaphylaxis; however, there is a scarcity of data in this regard.
\end{abstract}

Objective: This study described and characterized patients with urticaria, angioedema and type I hypersensitivity reactions caused by fibrinolytic agents.

Methods: This was a retrospective study in which cases of suspected adverse drug reactions from the use of streptokinase, alteplase, and tenecteplase were evaluated over a period of 10 years at Phramongkutklao and Ratchaburi hospitals in Thailand. In addition, patient characteristics and management were assessed.

Results: A total of 824 patients received fibrinolytic agents due to various indications. Of 147 patients who received streptokinase, nine $(6.12 \%)$ had suspected adverse drug reactions (one case of urticaria, two cases of anaphylactic shock, and six cases of hypotension). The prescription rate of alteplase was the highest, being taken by 547 patients; however, only one patient $(0.18 \%)$ reported an adverse reaction, angioedema in the face and lips. Similarly, of the 130 patients who received tenecteplase, only one patient $(0.77 \%)$ developed hypotension.

Conclusions: All fibrinolytic agents, either nonfibrin or fibrin-specific, can cause urticaria, angioedema, and type I hypersensitivity reactions due to their mechanism of action.

Key words: anaphylaxis, hypotension, streptokinase, tenecteplase, tissue plasminogen activator

\section{Citation:}

Duangmee, K., Boonmuang, P., Santimaleeworagun, W., Prasitdumrong, H. (2022). Urticaria, angioedema, and type I hypersensitivity reactions associated with fibrinolytic agents. Asian Pac J Allergy Immunol, 40(4), 379-385.

https://doi.org/10.12932/ap-050619-0574

\footnotetext{
Affiliations:

1 The College of Pharmacotherapy of Thailand, The Pharmacy Council, Nonthaburi, Thailand

${ }^{2}$ Department of Pharmacy, Faculty of Pharmacy, Silpakorn University, Nakhon Pathom, Thailand

Cardiovascular Unit, Department of Internal Medicine, Phramongkutklao Hospital, Bangkok, Thailand
}

Corresponding author:

Pornwalai Boonmuang

Faculty of Pharmacy, Silpakorn University,

Sanam Chandra Palace Campus

No.6 RajamankhaNai Road, PhraPathom Chedi, Muang,

Nakorn Pathom 10400, Thailand

E-mail: boonmuang_p@su.ac.th

\section{Introduction}

Type I or immediate-type hypersensitivity reactions, such as urticaria, angioedema, hypotension, and bronchospasm, generally occur within $24 \mathrm{~h}$ of exposure to an allergen. ${ }^{1}$ Although these reactions, including anaphylaxis, have been reported to have a low incidence, they may lead to life-threatening outcomes. The most common cause for these reactions is the use of several notorious medications, such as plasma components, angiotensin-converting enzyme inhibitors (ACEIs), penicillins and nonsteroidal antiinflammatory drugs (NSAIDs). ${ }^{2}$ Fibrinolytic agents can also induce allergic reactions, in addition to hemorrhage, thereby worsening clinical outcomes. Streptokinase, extracted from Group C beta-hemolytic streptococci, has been reported to be antigenic, activating antibodies in humans who receive it for the first time. ${ }^{3}$ Clinical trials have reported rates of hypersensitivity reactions and anaphylactic reactions associated with the use of streptokinase at $5 \%$ and $0.6 \%$, respectively. ${ }^{4-5}$ Unfortunately, these reactions have also been reported with the use of fibrin-specific fibrinolytic agents, 
such as tenecteplase and alteplase. The Global Utilization of Streptokinase and Tissue Plasminogen Activator for Occluded Coronary Arteries (GUSTO-I) trial showed that alteplase (1.5\%) was associated with a lower rate of hypersensitivity reactions than streptokinase; however, the rates of anaphylaxis were similar to those caused by tenecteplase..$^{5-8}$ Spontaneous reports of adverse drug reactions from the Thai Food and Drug Administration have described either urticaria, angioedema, or type I hypersensitivity reactions from the use of streptokinase and alteplase; however, adverse reactions caused by tenecteplase were assigned to an "unlikely" probability scale."

The clinical characteristics of patients who develop these hypersensitivity reactions still remain to be clearly defined, indicating further research is needed to determine the appropriate management. In this context, this study described all cases of urticaria, angioedema, and type I hypersensitivity reaction associated with the use of fibrinolytic agents for various indications at two hospitals in Thailand over a 10 year period, and also defined the characteristics of these patients.

\section{Methods}

We performed a retrospective search over a period of 10 years, from January 2008 through December 2017, of our databases at Phramongkutklao Hospital, a 1200-bed medical school hospital in Bangkok, and Ratchaburi Hospital, an 855-bed tertiary care hospital in Ratchaburi, Thailand. All patients who received streptokinase, alteplase, or tenecteplase were included in our study, using the individual medication codes of each hospital (Figure 1). Patients eligible for study inclusion were those diagnosed with acute myocardial infarction, acute ischemic heart disease, cerebral infarction, stroke, occlusion and stenosis of precerebral arteries, pulmonary embolism, and artery or vein thrombosis, according to ICD-10 definitions (I21, I24, I46, I63, I64, I65, I26, and I74, respectively). ${ }^{10}$ Exclusion criteria were unclear medical records regarding the use of fibrinolytic agents (e.g., incomplete records or missing data) and unspecified adverse drug reactions.

The protocol of our study was approved by the Institutional Review Board of the Royal Thai Army Medical Department, Phramongkutklao Hospital (approval number Q021h/61_Exp, issued on August 10, 2018) and Ratchaburi Hospital (COA-RBHEC005/2019, issued on January 18, 2019). The clinical trial registration number was TCTR20190607003, as approved by the Thai Clinical Trials Registry.

Medical records, either electronic or paper, were reviewed and patient information collected, including: gender; age; body weight; baseline systolic and diastolic blood pressure; comorbidities; medications prior to admission; history of medication allergies; history of prior streptokinase exposure, indications, dosage and administration, and premedication strategy; concomitant medications; echocardiograms; electrocardiograms; computed tomography (CT) scans; National Institutes of Health Stroke Scale (NIHSS) score; vital signs; and laboratory data. We identified urticaria, angioedema, or type I hypersensitivity reactions using the trigger words, "chlorpheniramine (CPM)" or "corticosteroid intravenous (IV) injection", and then specifically focused on the definitive diagnosis recorded by physicians for confirmation. The following additional details were extracted from the medical records: the first and the last dates of fibrinolytic agent use, the onset of suspected adverse drug reactions and its management, and the clinical outcome.

Urticaria was characterized by the appearance of pruritic, erythematous papules or plaques, with superficial swelling of the dermis. Angioedema was also defined by clinical manifestations, with bouts of asymmetric, independent swelling involving cutaneous or mucosal surfaces, for instance, the face, lips, tongue, oropharynx, extremities, or genitalia. ${ }^{1}$ Type I hypersensitivity reactions included anaphylactic reactions (anaphylaxis and anaphylactic shock), and hemodynamic (hypotension) and respiratory complications (e.g., bronchospasm, wheezes, dyspnea and chest discomfort). Anaphylaxis was confirmed when any one of the criteria of the National Institute of Allergy and Infectious Disease/Food Allergy and Anaphylaxis Network was fulfilled (Appendix A). ${ }^{11}$

All variables were analyzed using descriptive statistics, and data was managed by Microsoft Excel $^{\circledR}$ (Microsoft Corporation, Redmond, WA, USA). Medians with interquartile ranges (IQR) were determined, and these were used to obtain their percentages of demographic data.

\section{Results}

We identified 824 patients who received fibrinolytic agents due to various indications during the 10 year study period (Figure 1). Alteplase was the most frequently used medication, being used by 547 patients, of whom only one patient $(0.18 \%)$ reported angioedema in the face and lips. Streptokinase was prescribed to 147 patients, of whom nine $(6.12 \%)$ reported suspected adverse drug reactions. Tenecteplase use was detected in 130 patients, of whom only one patient $(0.77 \%)$ developed hypotension.

Table 1 shows the baseline characteristics of patients with suspected adverse drug reactions. Median age of the patients was 50 years (IQR 48-69) for the streptokinase group, and they were younger than patients who suffered reactions due to either alteplase (age 73 years) or tenecteplase (age 77 years). In the streptokinase group, $>88 \%$ of the patients were males, with a baseline body weight of $60 \mathrm{~kg}$ (IQR 58-78). The most common comorbidity was hypertension (4 patients, $44.44 \%$ ), followed by dyslipidemia (1 patient, 11.11\%) and diabetes mellitus (1 patient, 11.11\%).

The most common suspected adverse drug reaction due to streptokinase use was hypotension, which was reported in six of the nine patients. Two patients had anaphylactic shock, and one had urticaria; however, no case of angioedema was reported in this group. All patients had an acute myocardial infarction with no history of known drug allergies. Time-to-onset of the reaction to the medication ranged from $10 \mathrm{~min}$ to $4 \mathrm{~h}$; however, it generally occurred within an hour, and the median time was $28 \mathrm{~min}$. The management strategies applied to these patients are provided in Table 2. 


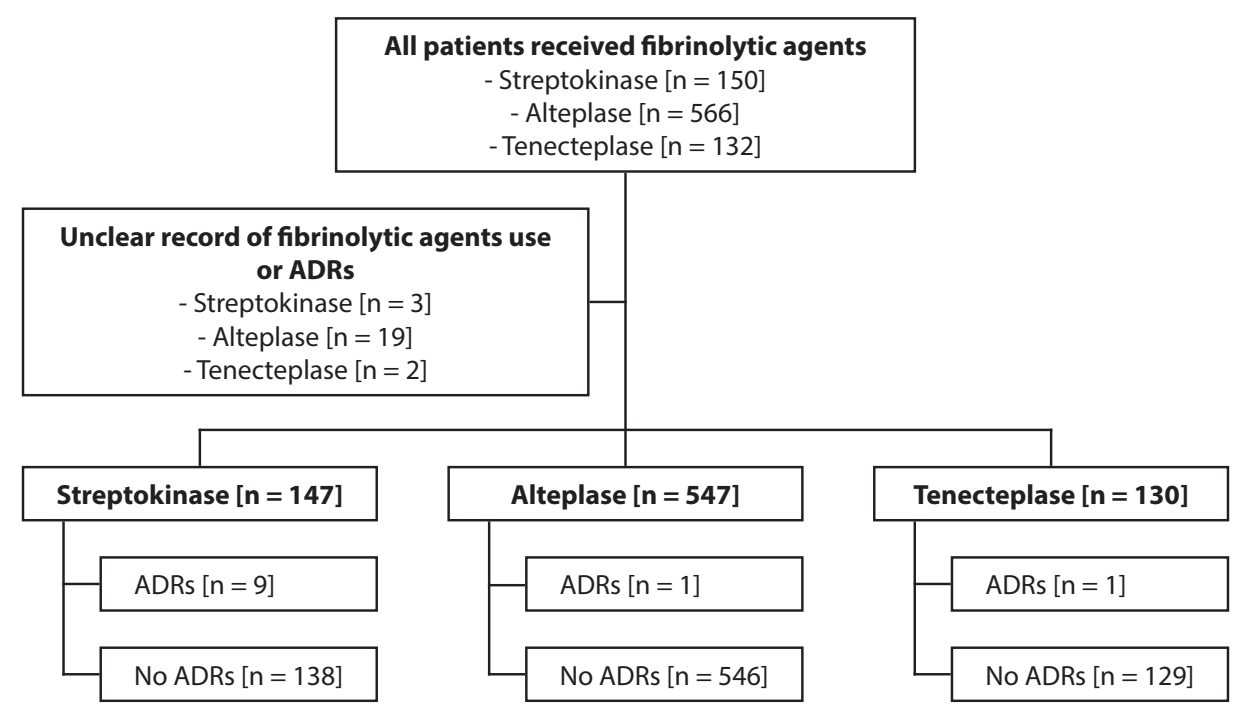

Figure 1. Selection of participants.

Table 1. Baseline characteristics of patients with fibrinolytic-associated urticaria, angioedema, and type I hypersensitivity reactions $s^{\S}$

\begin{tabular}{|c|c|c|c|}
\hline & Streptokinase $[\mathrm{n}=9]$ & Alteplase $[n=1]$ & Tenecteplase $[\mathrm{n}=1]$ \\
\hline Male (n) & 8 & 1 & 0 \\
\hline Median age (years) & 50 (IQR: 48, 69) & 73 & 77 \\
\hline Median body weight (kg) & 60 (IQR: 58, 78) & 65 & 75 \\
\hline \multicolumn{4}{|l|}{ Co-morbidities } \\
\hline Hypertension (n) & 4 & 1 & 1 \\
\hline Dyslipidemia (n) & 1 & 1 & 1 \\
\hline Diabetes mellitus (n) & 1 & 0 & 1 \\
\hline $\begin{array}{l}\text { Cerebrovascular } \\
\text { accident (n) }\end{array}$ & 0 & 1 & 0 \\
\hline \multicolumn{4}{|l|}{ Concomitant medications } \\
\hline ACEIs (n) & 1 & 1 & 0 \\
\hline ARBs (n) & 1 & 0 & 0 \\
\hline BBs (n) & 3 & 0 & 1 \\
\hline Aspirin (n) & 1 & 1 & 1 \\
\hline \multicolumn{4}{|l|}{ History of allergy } \\
\hline No known medicine allergy (n) & 9 & 1 & 1 \\
\hline \multicolumn{4}{|l|}{ Indications } \\
\hline $\begin{array}{l}\text { Acute myocardial infarction, } \\
\text { I } 21 \text { (n) }\end{array}$ & 9 & 0 & 1 \\
\hline Cerebral infarction, I63 (n) & 0 & 1 & 0 \\
\hline
\end{tabular}

ACEIs; angiotensin-converting enzyme inhibitors, ARBs; angiotensin-II receptor blockers, BBs; beta blockers, IQR; interquartile range ${ }^{5}$ Anaphylactic reactions (anaphylaxis, anaphylactic shock), hypotension, respiratory complications (e.g. wheeze, dyspnea, chest discomfort) 


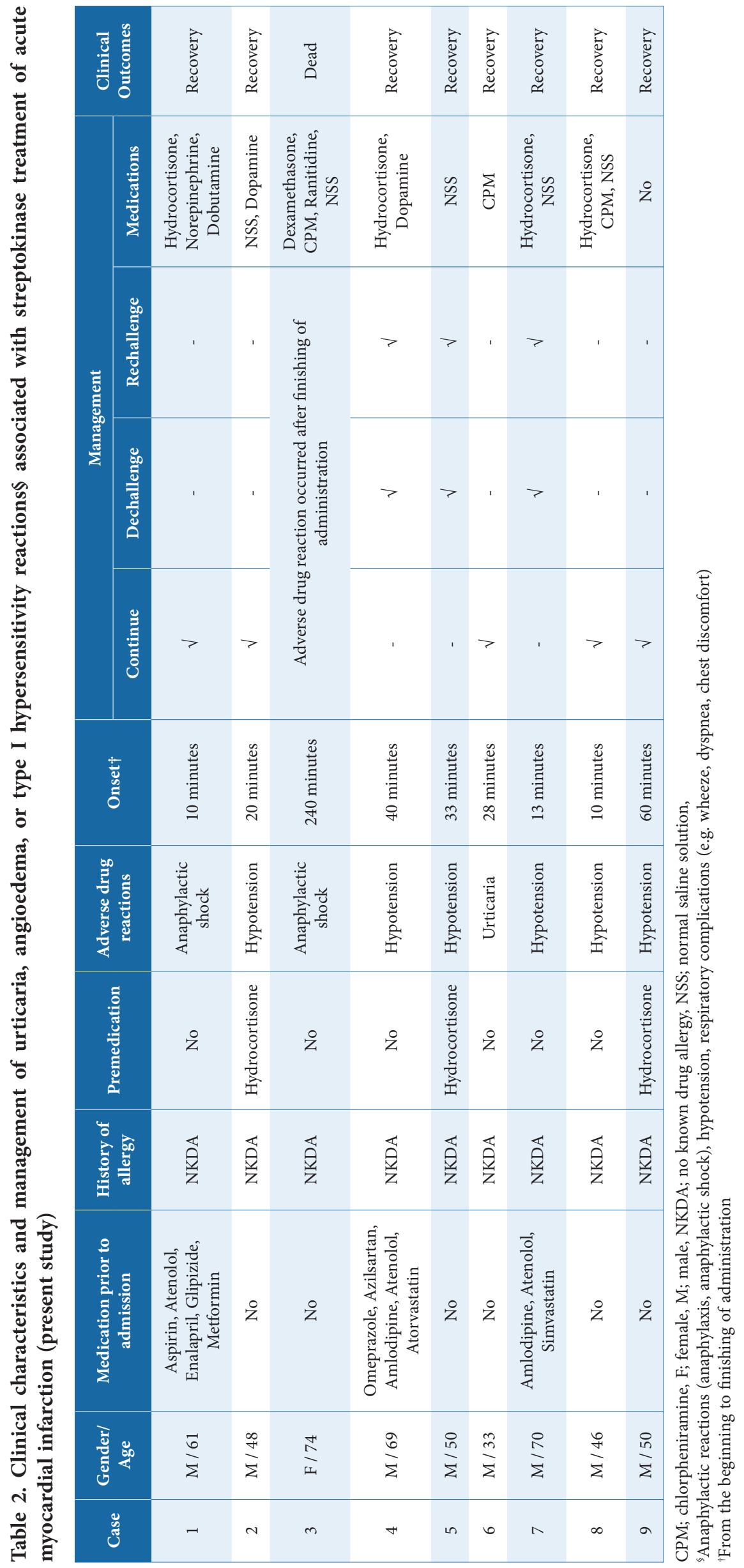


The patient who had angioedema in the face and lips due to being prescribed alteplase presented with right-sided hemiparesis and facial palsy. His previous medications included enalapril, amlodipine, aspirin, atorvastatin, omeprazole, and vitamin B complex. A CT scan of the brain revealed a hypodense lesion in the left middle cerebral artery, a dot sign, and loss of the cortical ribbon. Consequently, the physician diagnosed this case as acute ischemic stroke and he was started on $59 \mathrm{mg}$ alteplase. After $4 \mathrm{~h}$, his face and lips had swollen, and angioedema was diagnosed. The physician prescribed dexamethasone and an IV injection of CPM for management. His symptoms completely resolved over several hours.

Hypotension was the only suspected adverse drug reaction that was associated with tenecteplase use in this study. A 77-year-old woman was admitted to hospital with retrosternal chest pain that was subsequently confirmed to be caused by a ST-elevated myocardial infarction. She had also suffered from hypertension, dyslipidemia, and type 2 diabetes mellitus for several years, and her medications included low-dose aspirin, propranolol, cyproheptadine, and an extract of Curcuma longa. She also had a history of urticaria caused by simvastatin. In the emergency department, she was started on an IV bolus of $30 \mathrm{mg}$ tenecteplase over $5 \mathrm{~s}$, in addition to $300 \mathrm{mg}$ aspirin, $75 \mathrm{mg}$ clopidogrel, $75 \mathrm{mg}$ enoxaparin and $3 \mathrm{mg}$ morphine. After 6 min, her blood pressure had decreased from 106/72 to $86 / 54$ $\mathrm{mmHg}$, and subsequently to $62 / 44 \mathrm{mmHg}$. The physician prescribed an isotonic solution and dopamine for management, after which her blood pressure returned close to that of baseline level, at $105 / 63 \mathrm{mmHg}$.

For the streptokinase group, 11 of 147 patients (7.48\%) were administered an IV injection of corticosteroid as a premedication strategy. Six patients (54.54\%) received hydrocortisone, with two (18.18\%) receiving $100 \mathrm{mg}$ hydrocortisone and four patients (36.36\%) receiving $200 \mathrm{mg}$ hydrocortisone, while five patients $(45.45 \%)$ received $100 \mathrm{mg}$ hydrocortisone in combination with an IV injection of $10 \mathrm{mg}$ CPM, and one patient (9.09\%) received $4 \mathrm{mg}$ dexamethasone in combination with an IV injection of $10 \mathrm{mg}$ CPM. However, three patients (27.27\%) subsequently developed hypotension. For the tenecteplase group, only one of 130 patients $(0.77 \%)$ was administered premedication, an IV injection of $200 \mathrm{mg}$ hydrocortisone, and consequently they had no adverse drug reactions. By contrast, no premedication strategies were found for those taking alteplase.

\section{Discussion}

All fibrinolytic agents, nonfibrin and fibrin-specific, can induce hypersensitivity due to their mechanism of action, which activates the complement cascade and the kinin pathway, resulting in bradykinin congestion. ${ }^{12}$ Alteplase and tenecteplase generally result in a lower rate of adverse drug reactions than streptokinase, due to their specificity. Even so, several case reports involving adverse reactions to alteplase use have been published. However, similar studies of tenecteplase-related hypersensitivity reactions are rare.
The present study only found nine patients with suspected adverse drug reactions, among whom there was no case of angioedema, and only one patient of urticaria. History of a severe streptococcal infection or previous streptokinase exposure are well-known risk factors for anaphylaxis caused by streptokinase, ${ }^{13-15}$ while a rapid infusion rate has been reported to be a preventable risk factor for hypotension. ${ }^{16}$ We only found one patient who had a history of streptokinase exposure, seven years ago; however, no anaphylactic reaction occurred in this patient. Streptokinase-associated hypotension was found in this study, although all cases received the standard infusion rate over $60 \mathrm{~min}$. Misdiagnosis might be responsible for under-reporting of hypotension, as the symptoms were quite difficult to diagnose in patients with acute myocardial infarction, being easily confused with other clinical presentations, such as pulmonary edema or cardiogenic shock. ${ }^{17}$

Angiotensin-converting enzyme inhibitors (ACEIs) can inhibit plasma kininases that degraded bradykinin, although some patients with angioedema who were taking ACEIs might have a genetic defect associated with bradykinin metabolism. ${ }^{12}$ Hill et al. established some important data about the association between ACEIs and hemi-orolingual angioedema caused by alteplase, which was consistent with other studies; however, angiotensin-II receptor blockers (ARBs) were not associated. ${ }^{18-21}$ Cerebral ischemia in either the insular or the frontal cortex is another risk factor in addition to ACEIs; ${ }^{18-19}$ however, there are also contradictory reports. ${ }^{22}$ The present study only found one patient with angioedema in the face and lips caused by alteplase. This patient had previously received ACEIs and had an occlusion of the left middle cerebral artery. His clinical characteristics and management were also similar to those reported in previous studies; however, the major differences were the sites of angioedema and their slightly longer time in becoming evident. ${ }^{19-23}$

The current study provided new insights on type I hypersensitivity reactions, especially hypotension, associated with the use of tenecteplase. Previous controlled trials have not reported any specific case of hypersensitivity caused by tenecteplase, ${ }^{6-8}$ though Tebbi et al. reported one case of unspecified hypersensitivity reaction where tenecteplase was used because of a dysfunctional central venous catheter. ${ }^{24}$ The current study found a case of tenecteplase-associated hypotension. High-risk features were also examined, showing this patient also had akinesia in an extensive portion of the anterior wall of the heart, which was an important risk factor for hypotension. Nevertheless, the time-to-onset of hypotension was consistent with tenecteplase being causative. Despite the lack of antigenicity of teneecteplase due to amino acid sequence modifications, the abovementioned mechanism of bradykinin congestion due to the pharmacological action of fibrinolytic agents might be responsible for this reaction.

Potential evidence-based corticosteroid premedications are currently rarely described, and only small studies and case reports have been published recently; ${ }^{25-26}$ however, the mechanisms still remain unclear. A study of streptokinase 
use in pediatric patients with femoral artery obstruction reported that ranitidine and hydrocortisone might be effective in reducing the complications, which included hematoma and anaphylactic shock; however, the sample size in the report was small. ${ }^{27}$ In this study, three patients who subsequently developed hypotension after receiving streptokinase with a premedication strategy did not require additional corticosteroid treatment. However, patients with anaphylactic shock were not treated with any premedication. Therefore, corticosteroids may prevent severe hypersensitivity reactions and alleviate symptoms, though further studies are required to evaluate this issue.

No allergic reactions were reported in 4 and 35 patients with pulmonary embolism in the background of acute myocardial infarction who received streptokinase and alteplase, respectively, as a consequence of a slower infusion rate recommended by FDA-labeled dosage and administration guidelines. ${ }^{28}$ However, undistinguished signs or symptoms in these already symptomatic critically ill patients might be another reason.

The pattern of use of fibrinolytic agents in this current study was affected by several factors, such as indications, the type of hospital, national health insurance, and cost. As alteplase was the only fibrinolytic agent that was indicated for acute ischemic stroke, it was natural that most of participants in the current study received this medication for fibrinolytic therapy. Streptokinase, alteplase, and tenecteplase were indicated for acute myocardial infarction; however, the research sites were also hospitals with percutaneous coronary intervention (PCI) capabilities, meaning the use of fibrinolytic agents might not have been preferred. In addition, as the major healthcare coverage for the Thai population was universal coverage, tenecteplase was used at a lower rate than streptokinase because of a higher cost.

There were a few limitations in the current study. The retrospective design might have resulted in incomplete data. Data regarding pulmonary embolism and thromboembolectomies were rare, although it was dependent on the incidence of disease and clinical practice. Underreported adverse drug reactions might also be another limitation.

\section{Conclusion}

Fibrinolytic-associated urticaria, angioedema, and type I hypersensitivity reactions are potential and important complications requiring the attention of healthcare professionals, although most of the patients in this current study showed a complete recovery or did not have risk factors. As such, assessment of risk and monitoring of these allergic reactions should be performed carefully while initiating fibrinolytic therapy.

\section{Acknowledgments}

The authors declare no conflicts of interest associated with this research. No sources of funding were used to conduct this study or prepare this manuscript. Foremost, we gratefully acknowledge the computer center at Phramongkutklao Hospital for providing support on data access and their valuable inputs for the manuscript. The authors would like to thank Mr. Nirun Jangkong for gathering the database at Ratchaburi Hospital. Furthermore, we would like to thank Enago (www. enago.com) for the English language review.

\section{References}

1. Saini SS. Urticaria and Angioedema. In: Adkinson NF, editor. Middleton's Allergy: Principles and Practice. $8^{\text {th }}$ edition. Philadelphia: Elsevier Saunders; 2014. p. 575-87.

2. The International Collaborative Study of Severe Anaphylaxis. Risk of anaphylaxis in a hospital population in relation to the use of various drugs: an international study. Pharmacoepidemiol Drug Saf. 2003;12(3): 195-202.

3. Brogden RN, Speight TM, Avery GS. Streptokinase: a review of its clinical pharmacology, mechanism of action and therapeutic uses. Drugs. 1973;5(5):357-445.

4. ISIS-2 (Second International Study of Infarct Survival) Collaborative Group. Randomized trial of intravenous streptokinase, oral aspirin, both, or neither among 17,187 cases of suspected acute myocardial infarction: ISIS-2. Lancet. 1988;2(8607):349-60.

5. The GUSTO (Global Utilization of Streptokinase and Tissue Plasminogen Activator for Occluded Coronary Arteries) Investigators. An international randomized trial comparing four thrombolytic strategies for acute myocardial infarction. N Engl J Med. 1993;329(10): 673-82.

6. Van De Werf F, Cannon CP, Luyten A, Houbracken K, McCabe CH, Berioli S, et al. Safety assessment of single bolus administration of TNK-tPA in acute myocardial infarction: the ASSENT-I trial. Am Heart J. 1999;137(5):786-91.

7. Van De Werf F, Adgey J, Ardissino D, Armstrong PW, Aylward P, Barbash $\mathrm{G}$, et al. Single-bolus tenecteplase compared with frontloaded alteplase in acute myocardial infarction: the ASSENT-2 double-blind randomised trial. Lancet. 1999;354(9180):716-22.

8. The Assessment of the Safety and Efficacy of a New Thrombolytic Regimen (ASSENT)-3 Investigators. Efficacy and safety of tenecteplase in combination with enoxaparin, abciximab, or unfractionated heparin: the ASSENT-3 randomised trial in acute myocardial infarction. Lancet. 2001;358(9282):605-13.

9. HPVC. Spontaneous reports of adverse drug reactions 2016. Bangkok: Graphic and Design; 2016.

10. icd.who.int/en/ [Internet]. Geneva: International Classification of Disease 11th Revision; c2018 [cited by 2018 May 7]. Available from: https://icd. who.int/en/.

11. Sampson HA, Munoz-Furlong A, Campbell RL, Adkinson NF Jr, Bock SA, Branum A, et al. Second symposium on the definition and management of anaphylaxis: summary report--Second National Institute of Allergy and Infectious Disease/Food Allergy and Anaphylaxis Network symposium. J Allergy Clin Immunol. 2006;117(2):391-7.

12. Hill MD, Barber PA, Takahashi J, Demchuk AM, Feasby TE, Buchan AM. Anaphylactoid reactions and angioedema during alteplase treatment of acute ischemic stroke. CMAJ. 2000;162(9):1281-4.

13. Fears R, Ferres H, Glasgow E, Standring R, Hogg KJ, Gemmill JD, et al. Monitoring of streptokinase resistance titre in acute myocardial infarction patients up to 30 months after giving streptokinase or anistreplase and related studies to measure specific antistreptokinase IgG. Br Heart J. 1992;68(2):167-70.

14. Squire IB, Lawley W, Fletcher S, Holme E, Hillis WS, Hewitt C, et al. Humoral and cellular immune responses up to 7.5 years after administration of streptokinase for acute myocardial infarction. Eur Heart J. 1999;20(17):1245-52.

15. Nitzsche R, Rosenheinrich M, Kreikemeyer B, Oehmcke-Hecht S. Streptococcus pyogenes triggers activation of the human contact system by streptokinase. Infect Immun. 2015;83(8):3035-42. 
16. Lew AS, Laramee P, Cercek B, Shah PK, Ganz W. The hypotensive effect of intravenous streptokinase in patients with acute myocardial infarction. Circulation. 1985;72(6):1321-6.

17. David CS, Robert MC. Complications of Thrombolytic Therapy. In: Eric RB, editor. Thrombolysis and Adjunctive Therapy for Acute Myocardial Infarction. New York: Marcel Dekker; 1993. p. 91-115.

18. Hill MD, Lye T, Moss H, Barber PA, Demchuk AM, Newcommon NJ, et al. Hemi-orolingual angioedema and ACE inhibition after alteplase treatment of stroke. Neurology. 2003;60(9):1525-7.

19. Myslimi F, Caparros F, Dequatre-Ponchelle N, Moulin S, Gautier S, Girardie P, et al. Orolingual angioedema during or after thrombolysis for cerebral ischemia. Stroke. 2016;47(7):1825-30.

20. Lin SY, Tang SC, Tsai LK, Yeh SJ, Hsiao YJ, Chen YW, et al. Orolingual angioedema after alteplase therapy of acute ischaemic stroke: incidence and risk of prior angiotensin-converting enzyme inhibitor use. Eur J Neurol. 2014;21(10):1285-91.

21. Hurford R, Rezvani S, Kreimei M, Herbert A, Vail A, Parry-Jones AR, et al. Incidence, predictors and clinical characteristics of orolingual angio-oedema complicating thrombolysis with tissue plasminogen activator for ischaemic stroke. J Neurol Neurosurg Psychiatry. 2015; 86(5):520-3.

22. Pinho J, Alves JN, Oliveira L, Pereira S, Barros J, Machado C, et al. Orolingual angioedema after thrombolysis is not associated with insular cortex ischemia on pre-thrombolysis CT. J Neurol Sci. 2016;369:48-50.
23. Correia AS, Matias G, Calado S, Lourenco A, Viana-Baptista M. Orolingual angioedema associated with alteplase treatment of acute stroke: a reappraisal. J Stroke Cerebrovasc Dis. 2015;24(1):31-40.

24. Tebbi C, Costanzi J, Shulman R, Dreisbach L, Jacobs BR, Blaney M, et al. A phase III, open-label, single-arm study of tenecteplase for restoration of function in dysfunctional central venous catheters. J Vasc Interv Radiol. 2011;22(8):1117-23.

25. McGrath KG, Patterson R. Anaphylactic reactivity to streptokinase. JAMA. 1984;252(10):1314-7.

26. Dykewicz MS, McGrath KG, Davison R, Kaplan KJ, Patterson R. Identification of patient at risk for anaphylaxis due to streptokinase. Arch Intern Med. 1986;146(2):305-7.

27. Noori N, Miri Aliabad G, Mohammadi M, Mahjoubifard M, Jahangiri Fard A. The effects of ranitidine and hydrocortisone on the complications of femoral artery obstruction treated by streptokinase following cardiac catheterization in pediatric patients with congenital heart diseases. Iran Red Crescent Med J. 2013;15(2):117-21.

28. Konstantinides SV, Torbicki A, Agnelli G, Danchin N, Fitzmaurice D, Galie N, et al. 2014 ESC guidelines on the diagnosis and management of acute pulmonary embolism: The Task Force for the Diagnosis and Management of Acute Pulmonary Embolism of the European Society of Cardiology (ESC). Eur Heart J. 2014;35(43):3033-69. 